\title{
TENSOR PRODUCT MAPPINGS. II
}

\author{
J. R. HOLUB ${ }^{1}$
}

\begin{abstract}
In this paper factorization techniques are introduced into the study of tensor product mappings to complete and improve on some results obtained by the author in an earlier paper [Tensor product mappings, Math. Ann. 188 (1970), 1-12. MR 44 \#2052]. The main results are as follows: Let $\alpha$ be any $\otimes$-norm. Then

(i) if $S$ is absolutely summing and $T$ is an integral operator then $S \otimes_{\alpha} T$ is absolutely summing,

(ii) if $S$ is quasi-nuclear and $T$ is nuclear then $S \otimes_{\alpha} T$ is quasinuclear,

(iii) if $S$ and $T$ are integral operators then $S \otimes_{\alpha} T$ is integral.

That the results (i) and (ii) are essentially the best possible was shown by examples in the earlier quoted paper. Also, the methods developed in this paper yield a much simpler proof of the main result of the earlier paper.
\end{abstract}

The purpose of this paper is to continue the investigation of tensor product mappings begun in [4] and to demonstrate the use of factorization techniques in this study. In [4] various permanence properties of certain classes of operators were proved and contrasts in the results obtained between the two crossnorms $\varepsilon$ and $\pi$ on the tensor product were discussed. In particular it was shown that if $S$ and $T$ are absolutely summing operators (resp., quasi-nuclear operators) then $S \otimes_{\varepsilon} T$ is absolutely summing (resp., quasi-nuclear). However, simple examples show that for each of these cases $S \otimes_{\bar{\pi}} T$ need not have the property. In this paper we show that by simply requiring one of $S$ or $T$ to satisfy a slightly stronger condition we can achieve permanence of the above properties for any $\otimes$-norm [2] on the tensor product. More precisely,

(i) if $S$ is absolutely summing and $T$ is an integral operator [1] then $S \otimes_{\alpha} T$ is absolutely summing for every $\otimes$-norm $\alpha$;

(ii) if $S$ is a quasi-nuclear and $T$ is nuclear then $S \otimes_{\alpha} T$ is quasi-nuclear for every $\otimes-$ norm $\alpha$.

Received by the editors May 10, 1973.

AMS (MOS) subject classifications (1970). Primary 47B10.

Key words and phrases. Tensor products, absolutely summing operator, integral operator, nuclear operator, factorization of operators.

${ }^{1}$ The research for this paper was partially supported by NSF-GP 33778.

(C) American Mathematical Society 1974 
An examination of the proof of (i) also establishes

(iii) if $S$ and $T$ are integral operators then $S \otimes_{\alpha} T$ is integral for every $\otimes$-norm $\alpha$.

In addition to these results we give a greatly simplified proof of the main theorem of [4].

Our results depend upon the introduction of factorization techniques into the study of tensor product mappings and upon known factorization theorems for the classes of operators we study. The simplicity of this viewpoint in the investigation of certain problems connected with tensor product mappings will, it is hoped, be amply demonstrated.

Throughout the paper $\dot{E}_{\mathrm{i}}, E_{2}, F_{1}$ and $F_{2}$ will denote Banach spaces and the term "operator" or "map" will always refer to a continuous linear transformation. We often indicate that $A$ is an operator from $E$ to $F$ by $E \rightarrow{ }^{\wedge} F$. Any unexplained notation or concept will be as in [4]. We also refer the reader to this paper for the necessary background information concerning the classes of operators under discussion.

Since it does not appear in [4] but is crucial to our work we give here the definition of an integral operator (see [1] for a complete discussion of this notion).

Definition. An operator $T: E \rightarrow F$ is said to be integral if $T$ has the factorization

$$
T: E \longrightarrow C(K) \stackrel{i}{\longrightarrow} L^{1}(\mu) \longrightarrow F^{* *},
$$

where $K$ is the unit ball in $E^{*}, \mu$ is a Borel measure on $K$, and $i$ is the injection map.

It is well known that every integral map is absolutely summing [1], [9], and that an absolutely summing map whose range is in $\ell^{\infty}(\Gamma)$ for some set $\Gamma$ is integral [10].

THEOREM 1. Let $S: E_{1} \rightarrow E_{2}$ be an absolutely summing operator and $T: F_{1} \rightarrow F_{2}$ an integral operator. Then $S \otimes_{\alpha} T: E_{1} \otimes_{\alpha} F_{1} \rightarrow E_{2} \otimes_{\alpha} F_{2}$ is absolutely summing for any $\otimes$-norm $\alpha$.

Proof. Let $j: E_{2} \rightarrow \ell^{\infty}(\Gamma)$ be the natural isometry of $E_{2}$ into the space of bounded functions on the unit ball $\Gamma$ in $E_{2}^{*}$. Then from the remarks above $j \circ S$ is integral and hence has the factorization

$$
j \circ S: E_{1} \stackrel{A_{1}}{\longrightarrow} C\left(K_{1}\right) \stackrel{i_{1}}{\longrightarrow} L^{1}(\mu) \stackrel{B_{1}}{\longrightarrow} C^{\infty}(\Gamma) .
$$

Similarly, since $T$ is integral it can be written

$$
T: F_{1} \stackrel{A_{2}}{\longrightarrow} C\left(K_{2}\right) \stackrel{i_{2}}{\longrightarrow} L^{1}(v) \stackrel{B_{2}}{\longrightarrow} F_{2}^{* *} .
$$


Let $Q=i_{2} \circ A_{2}$ and consider the mapping $(j \circ S) \otimes Q$. Clearly this map factors as

$$
\begin{aligned}
(j \circ S) \otimes Q: E_{1} \otimes_{\alpha} F_{1} \stackrel{A_{1} \otimes_{\alpha} A_{2}}{\longrightarrow} & C\left(K_{1}\right) \otimes_{\alpha} C\left(K_{2}\right) \\
\stackrel{i_{1} \otimes i_{2}}{\longrightarrow} & L^{1}(\mu) \otimes_{\alpha} L^{1}(v) \stackrel{B_{1} \otimes I}{\longrightarrow} \ell^{\infty}(\Gamma) \otimes_{\alpha} L^{1}(v),
\end{aligned}
$$

where $I$ denotes the identity operator.

Recall that $\varepsilon \leqq \alpha \leqq \pi$ and that $C\left(K_{1}\right) \otimes_{\varepsilon} C\left(K_{2}\right)=C\left(K_{1} \times K_{2}\right)$, while $L^{1}(\mu) \otimes_{\pi} L^{1}(v)=L^{1}(\mu \times v)$ [1]. Thus the operator $\left(B_{1} \otimes I\right) \circ\left(i_{1} \otimes i_{2}\right)$ factors as

$$
\begin{aligned}
&\left(B_{1} \otimes I\right) \circ\left(i_{1} \otimes i_{2}\right): C\left(K_{1}\right) \otimes_{\alpha} C\left(K_{2}\right) \longrightarrow C\left(K_{1}\right) \otimes_{\varepsilon} C\left(K_{2}\right) \\
& \stackrel{i}{\longrightarrow} L^{1}(\mu) \otimes_{\pi} L^{1}(v) \stackrel{B_{1} \otimes I}{\longrightarrow} \ell^{\infty}(\Gamma) \otimes_{\pi} L^{1}(v),
\end{aligned}
$$

where $i$ is the injection of $C\left(K_{1} \times K_{2}\right)$ into $L^{1}(\mu \times v)$ and is integral. This implies that $(j \circ S) \otimes Q: E_{1} \otimes_{\alpha} F_{1} \rightarrow \ell^{\infty}(\Gamma) \otimes_{\pi} L^{1}(v)$ is integral, hence absolutely summing. Since clearly it also factors as

$$
(j \circ S) \otimes Q: E_{1} \otimes_{\alpha} F_{1} \longrightarrow E_{2} \otimes_{\pi} L^{1}(v) \stackrel{j \otimes_{\pi} I}{\longrightarrow} \ell^{\infty}(\Gamma) \otimes_{\pi} L^{1}(v),
$$

where $j \otimes_{\pi} I$ is an isometry [3], it follows that $S \otimes Q: E_{1} \otimes_{\alpha} F_{1} \rightarrow$ $E_{2} \otimes_{\pi} L^{1}(v)$ is also absolutely summing, as is $S \otimes T=\left(I \otimes B_{2}\right) \circ(S \otimes Q)$ : $E_{1} \otimes_{\alpha} F_{1} \rightarrow E_{2} \otimes_{\pi} F_{2}^{* *}$. Since $E_{2} \otimes_{\pi} F_{2}$ is isometrically embedded in $E_{2} \otimes_{\pi} F_{2}^{* *}$ [1] we must have that $S \otimes T: E_{1} \otimes_{\alpha} F_{1} \rightarrow E_{2} \otimes_{\pi} F_{2}$ is an absolutely summing operator. But then certainly $S \otimes_{\alpha} T: E_{1} \otimes_{\alpha} F_{1} \rightarrow E_{2} \otimes_{\alpha} F_{2}$ is also absolutely summing (since $\alpha \leqq \pi$ ).

Simple modifications of the proof of Theorem 1 also establish the following two results.

THEOREM 2. If $S: E_{1} \rightarrow E_{2}$ and $T: F_{1} \rightarrow F_{2}$ are both integral operators then $S \otimes_{\alpha} T: E_{1} \otimes_{\alpha} F_{1} \rightarrow E_{2} \otimes_{\alpha} F_{2}$ is an integral operator for any $\otimes$-norm $\alpha$.

THEOREM 3. If $S: E_{1} \rightarrow L^{1}(\mu)$ and $T: F_{1} \rightarrow F_{2}$ are both absolutely summing then $S \otimes_{\alpha} T: E_{1} \otimes_{\alpha} F_{1} \rightarrow L^{1}(\mu) \otimes_{\alpha} F_{2}$ is absolutely summing for any $\otimes$-norm $\alpha$.

We now apply a similar technique to show that the tensor product of a quasi-nuclear and a nuclear map is quasi-nuclear.

THEOREM 4. Let $S: E_{1} \rightarrow E_{2}$ be a quasi-nuclear operator and $T: F_{1} \rightarrow F_{2}$ a nuclear operator. Then $S \otimes_{\alpha} T: E_{1} \otimes_{\alpha} F_{1} \rightarrow E_{2} \otimes_{\alpha} F_{2}$ is quasi-nuclear for any $\otimes$-norm $\alpha$.

Proof. As in the proof of Theorem 1, let $j: E_{2} \rightarrow \ell^{\infty}(\Gamma)$ be the canonical isometry. Since $S$ is quasi-nuclear the mapping $j \circ S: E_{1} \rightarrow \ell^{\infty}(\Gamma)$ is nuclear 
[8] and hence can be factored as

$$
j \circ S: E_{1} \stackrel{A_{1}}{\longrightarrow} c_{0} \stackrel{a_{1}}{\longrightarrow} \ell^{1} \stackrel{B_{1}}{\longrightarrow} \ell^{\infty}(\Gamma),
$$

where $q_{1}$ is the nuclear operator defined by $q_{1}\left(c_{i}\right)=\left(a_{i} c_{i}\right)$ for some $\left(a_{i}\right) \in \ell^{1}[10]$.

Similarly $T$, being nuclear, can be factored as

$$
T: E_{2} \stackrel{A_{2}}{\longrightarrow} c_{0} \stackrel{a_{2}}{\longrightarrow} \ell^{1} \stackrel{\mathrm{B}_{2}}{\longrightarrow} F_{2},
$$

where $q_{2}$ is defined by $q_{2}\left(c_{i}\right)=\left(b_{i} c_{i}\right)$ for $\left(b_{i}\right) \in \ell^{1}$.

Let $R=q_{2} \circ A_{2}: E_{2} \rightarrow \ell^{1}$. Then $(j \circ S) \otimes R$ has the factorization

$$
\begin{aligned}
(j \circ S) \otimes R: E_{1} \otimes_{\alpha} F_{1} \stackrel{A_{1} \otimes A_{2}}{\longrightarrow} c_{0} \otimes_{\alpha} c_{0} \\
\stackrel{a_{1} \otimes a_{2}}{\longrightarrow} \ell^{1} \otimes_{\alpha} \ell^{1} \stackrel{B_{1} \otimes I}{\longrightarrow} \ell^{\infty}(\Gamma) \otimes_{\alpha} \ell^{1} .
\end{aligned}
$$

Now $q_{1} \otimes q_{2}$ is the mapping defined by $q_{1} \otimes q_{2}\left(e_{i} \otimes e_{j}\right)=a_{i} b_{j} e_{i} \otimes e_{j}$ (where $\left(e_{i}\right)$ denotes the unit vector basis for $c_{0}$ and for $\ell^{1}$ ). One checks easily that $q_{1} \otimes q_{2}$ actually takes $c_{0} \otimes_{\varepsilon} c_{0}$ into $\ell^{1} \otimes_{\pi} \ell^{1}$ and is nuclear. Hence we have the factorization

$$
q_{1} \otimes q_{2}: c_{0} \otimes_{\alpha} c_{0} \rightarrow c_{0} \otimes_{c} c_{0} \rightarrow \ell^{1} \otimes_{\pi} l^{1} \rightarrow \ell^{1} \otimes_{\alpha} \ell^{1},
$$

implying that $(j \circ S) \otimes R$ is nuclear, having the factorization

$$
\begin{aligned}
&(j \circ S) \otimes R: E_{1} \otimes_{\alpha} F_{1} \longrightarrow c_{0} \otimes_{\alpha} c_{0} \longrightarrow c_{0} \otimes_{\varepsilon} c_{0} \\
& \stackrel{\varphi}{\longrightarrow} \ell^{1} \otimes_{\pi} \ell^{1} \stackrel{B_{1}(j) I}{\longrightarrow} l^{\infty}(\Gamma) \otimes_{\pi} \ell^{1},
\end{aligned}
$$

where $Q$ is nuclear. As in the proof of Theorem 1 , since $j$ is an isometry we conclude that $E_{2} \otimes_{\pi} \ell^{1}$ is isometric to a subspace of $\ell^{\infty}(\Gamma) \otimes_{\pi} \ell^{1}$ [3] and hence the mapping $S \otimes R: E_{1} \otimes_{\alpha} F_{1} \rightarrow E_{2} \otimes_{\pi} / 1$ is quasi-nuclear.

But then $S \otimes T=\left(I \otimes B_{2}\right) \circ(S \otimes R): E_{1} \otimes_{\alpha} F_{1} \rightarrow E_{2} \otimes_{\pi} F_{2}$ is quasi-nuclear, implying $S \otimes_{\alpha} T: E_{1} \otimes_{\alpha} F_{1} \rightarrow E_{2} \otimes_{\alpha} F_{2}$ is also quasi-nuclear.

Again, a slight modification of the proof of Theorem 4 shows

THEOREM 5. Let $S: E_{1} \rightarrow L^{1}(\mu)$ and $T: F_{1} \rightarrow F_{2}$ be quasi-nuclear operators. Then $S \otimes_{\alpha} T$ is quasi-nuclear for every $\otimes-n o r m \alpha$.

Finally, using the techniques demonstrated above we give a much simpler proof of the following result which was proved in [4]:

If $S: E_{1} \rightarrow E_{2}$ and $T: F_{1} \rightarrow F_{2}$ are $p$-absolutely summing operators then $S \otimes_{\varepsilon} T: E_{1} \otimes_{\varepsilon} F_{1} \rightarrow E_{2} \otimes_{\varepsilon} F_{2}$ is p-absolutely summing.

Proof. Let $\Gamma_{1}$ and $\Gamma_{2}$ denote the unit balls in $E_{2}^{*}$ and $F_{2}^{*}$, respectively. Then, as above, let $j_{1}: E_{2} \rightarrow l^{x}\left(\Gamma_{1}\right)$ and $j_{2}: F_{2} \rightarrow \ell^{x}\left(\Gamma_{2}\right)$ be the canonical isometries. Since $S$ and $T$ are $p$-absolutely summing it follows easily 
from [6] and [5] that we can write

$$
\begin{aligned}
& j \circ S: E_{1} \longrightarrow C\left(K_{1}\right) \stackrel{i_{1}}{\longrightarrow} L^{p}(\mu) \longrightarrow \ell^{\infty}\left(\Gamma_{1}\right), \\
& j_{2} \circ T: F_{1} \longrightarrow C\left(K_{2}\right) \stackrel{i_{2}}{\longrightarrow} L^{p}(v) \longrightarrow \ell^{\infty}\left(\Gamma_{2}\right),
\end{aligned}
$$

where $K_{1}$ and $K_{2}$ are the unit balls in $E_{1}^{*}$ and $F_{1}^{*}$ and $i_{1}$ and $i_{2}$ are injection maps.

It follows that

$$
\begin{aligned}
\left(j_{1} \circ S\right) \otimes_{\varepsilon}\left(j_{2} \circ T\right): E_{1} \otimes_{\varepsilon} E_{2} & \longrightarrow C\left(K_{1}\right) \otimes_{\varepsilon} C\left(K_{2}\right) \\
\stackrel{i_{1} \otimes i_{2}}{\longrightarrow} & L^{p}(\mu) \otimes_{\varepsilon} L^{p}(v) \longrightarrow \ell^{\infty}\left(\Gamma_{1}\right) \otimes_{\varepsilon} \ell^{\infty}\left(\Gamma_{2}\right) .
\end{aligned}
$$

Let $\alpha_{p}$ denote the crossnorm on $L^{p}(\mu) \otimes L^{p}(v)$ for which

$$
L^{p}(\mu) \otimes_{\alpha_{p}} L^{p}(v)=L^{p}(\mu \times v) .
$$

Then $i_{1} \otimes i_{2}$ factors as

$$
i_{1} \otimes i_{2}: C\left(K_{1}\right) \otimes_{\varepsilon} C\left(K_{2}\right) \stackrel{j}{\rightarrow} L^{p}(\mu) \otimes_{\alpha_{p}} L^{p}(v) \longrightarrow L^{p}(\mu) \otimes_{\varepsilon} L^{p}(v),
$$

recalling that $C\left(K_{1}\right) \otimes_{\varepsilon} C\left(K_{2}\right)=C\left(K_{1} \times K_{2}\right)$ and $j$ is the injection map of $C\left(K_{1} \times K_{2}\right)$ into $L^{p}(\mu \times v)$, a $p$-absolutely summing operator [7]. It follows that $\left(j_{1} \circ S\right) \otimes_{\varepsilon}\left(j_{2} \circ T\right)$ is $p$-absolutely summing. But since $E_{2} \otimes_{\varepsilon} F_{2}$ is isometrically embedded in $\ell^{\infty}\left(\Gamma_{1}\right) \otimes_{\varepsilon} \ell^{\infty}\left(\Gamma_{2}\right)$ it then follows that $S \otimes_{c} T$ is also $p$-absolutely summing.

\section{REFERENCES}

1. A. Grothendieck, Produits tensoriels topologiques et espaces nucléaires, Mem. Amer. Math. Soc. No. 16 (1955). MR 17, 763.

2. - Résumé de la théorie métrique des produits tensoriels topologiques, Bol. Soc. Mat. São Paulo 8 (1953), 1-79. (1956). MR 20 \#1194.

3. Une caractérisation vectorielle-métrique des espaces $L^{1}$, Canad. J. Math. 7 (1955), 552-561. MR 17, 877.

4. J. R. Holub, Tensor product mappings, Math. Ann. 188 (1970), 1-12. MR 44 \#2052.

5. J. Lindenstrauss, Extension of compact operators, Mem. Amer. Math. Soc. No. 48 (1964). MR 31 \#3828.

6. J. Lindenstrauss and A. Pelczyński, Absolutely summing operators in $\mathscr{L}_{p}$-spaces and their applications, Studia Math 29 (1968), 275-326. MR 37 \#6743.

7. A. Pietsch, Absolut p-summierende Abbildungen in normierten Raümen, Studia Math. 28 (1966/67), 333-353. MR 35 \#7162.

8. - Quasinukleare Abbildungen in normierten Raümen, Math. Ann. 165 (1966), 76-90. MR 33 \#6412.

9. A. Pietsch and A. Perrson, p-nukleare and p-integrale Abbildungen in Banach Raümen, Studia Math 33 (1969), 19-62. MR 39 \#4645.

10. C. Stegall and J. R. Retherford, Fully nuclear and completely nuclear operators with applications to $\mathscr{L}_{1}$ and $\mathscr{L}_{\infty}$-spaces, Trans. Amer. Math. Soc. 163 (1972), 457-492.

Department of Mathematics, Virginia Polytechnic Institute and State UNIVERSTTY, BLACKSBURG, VIRGINIA 24061 